\title{
Kinetics and design of distillation reactor with ZSM-5@SiC foam catalytic packing for EL synthesis in industrial scale
}

\author{
Qiuyan Ding ${ }^{1}$, Hong $\mathrm{Li}^{1}$, Zihao Chen ${ }^{1}$, Yanan Guan ${ }^{2}$, Xingang $\mathrm{Li}^{1}$, Jinsong Zhang ${ }^{2}$, Yilai \\ $\mathrm{Jiao}^{2}$, Jing Zou ${ }^{1}$, and Xin $\mathrm{Gao}^{3}$ \\ ${ }^{1}$ Tianjin University \\ ${ }^{2}$ Chinese Academy of Sciences \\ ${ }^{3}$ Tianjin university
}

March 6, 2021

\begin{abstract}
Distillation reactor is one of the important equipment to improve the reaction conversion limited by the chemical equilibrium, however, the design of distillation reactor in industrial scale is still challenging due to the lack of efficient catalytic packing with corresponding kinetics. This work developed the novel catalytic packing consists of the structured ZSM-5@SiC foam catalyst and corrugated sheet, which used in distillation reactor for synthesis of ethyl levulinate (EL). The kinetic of structured ZSM$5 @ \mathrm{SiC}$ foam catalyst was measured and model regarding the "structure factor" of ZSM-5@SiC catalyst and fluid flow conditions. Furthermore, the design of distillation reactor in industrial scale has been performed based on the developed kinetics, which demonstrated an excellent performance in the designed distillation reactor (EL yield 37.65\% with energy consumption 3695 $\mathrm{kJ} / \mathrm{kg}$ ) are achieved. X-ray CT characterization revealed that the ZSM-5 coating thickness has the significantly effects on the kinetics and performances of distillation reactor.
\end{abstract}

\section{Hosted file}

Manuscript.pdf available at https://authorea.com/users/399966/articles/512363-kinetics-anddesign-of-distillation-reactor-with-zsm-5-sic-foam-catalytic-packing-for-el-synthesisin-industrial-scale 